\title{
Evaluation of Carbapenem-Resistant
}

Enterobacteriaceae (CRE) Guideline Implementation in the Veteran's Affairs Medical Centers Using the Consolidated Framework for Implementation Research

Cassie Cunningham Goedken ( $\nabla$ cassie.goedken@va.gov )

lowa City VA Medical Center https://orcid.org/0000-0003-0990-1799

Marylou Guihan

Edward Hines Junior VA Hospital

Charnetta R. Brown

Eagle Hill Consulting

Swetha Ramanathan

Edward Hines Junior VA Hospital

Amanda Vivo

Edward Hines Junior VA Hospital

Katie J. Suda

VA Pittsburgh Healthcare System

Margaret A. Fitzpatrick

Edward Hines Junior VA Hospital

Linda Poggensee

Edward Hines Junior VA Hospital

Eli N. Perencevich

Iowa City VA Medical Center

Michael Rubin

VA Salt Lake City Health Care System

Heather Schacht Reisinger

lowa City VA Medical Center

Martin Evans

Lexington VA Medical Center

Charlesnika T. Evans

Edward Hines Junior VA Hospital 
Keywords: Carbapenem-resistant Enterobacteriaceae (CRE), implementation science, Consolidated Framework for Implementation Research (CFIR), Multi-drug Resistant Organisms (MDROs)

Posted Date: August 25th, 2020

DOI: https://doi.org/10.21203/rs.3.rs-54590/v1

License: (c) (1) This work is licensed under a Creative Commons Attribution 4.0 International License. Read Full License 


\section{Abstract}

Background Infections caused by carbapenem-resistant Enterobacteriaceae (CRE) and carbapenemaseproducing (CP) CRE are difficult to treat, resulting in high mortality in healthcare settings every year. The Veteran's Health Administration (VHA) disseminated guidelines in 2015 and an updated directive in 2017 for control of CRE focused on laboratory testing, prevention, and management. The Consolidated Framework for Implementation Research (CFIR) framework was used to examine contextual factors and identify best practices influencing implementation of the 2015 guidelines/2017 directive in VA Medical Centers (VAMCs).

Methods Qualitative interviews were conducted at 29 VAMCs with staff involved in implementing CRE guidelines at their facility. Survey and VHA administrative data were used to identify geographically representative large and small VAMCs with varying levels of CRE incidence. Interviews addressed perceptions of guideline dissemination, laboratory testing, staff attitudes and training, patient education, and technology support. Participant responses were coded using a consensus-based mixed deductiveinductive approach guided by CFIR. A quantitative analysis comparing CFIR constructs and emergent codes to sites actively screening for CRE (vs. non-screening) and any (vs. no) CRE positive cultures was conducted with a Fisher's exact test.

Results Forty-three semi-structured interviews were conducted between 10/2017-8/2018 with laboratory staff (47\%), Multi-Drug Resistant Organism Program Coordinators (MPCs, 35\%), infection preventionists (12\%), and physicians (6\%). Participants cited the need for more standardized tools to promote effective communication (e.g., electronic screening). Participants also indicated that CRE-specific educational materials were needed for staff, patient, and family members. Quantitative analysis identified the following CFIR constructs as being significantly associated with CRE screening or presence of CRE: Leadership Engagement, Relative Priority Available Resources, Team Communication, and Access to Knowledge \& Information.

Conclusions Effective CRE identification, prevention and treatment requires ongoing collaboration between clinical, microbiology, infection prevention, antimicrobial stewardship, and infectious diseases specialists. Our results emphasize the importance of leadership's role in promoting positive facility culture, including increasing healthcare worker's awareness of infection control and prevention for emerging pathogens, access to resources, improving communication and facilitating successful implementation of the CRE guidelines.

\section{Contributions To The Literature}

- Implementing guidelines to prevent emerging pathogens builds on strong facility-level culture and infection control practices.

- Our findings suggest access to available resources, effective communication tools, engaged leadership, and strong infection control infrastructure facilitate successful implementation of CRE 
guidelines.

- Our findings contribute to the implementation literature by emphasizing the importance of local leadership's role in providing access to available resources as well as promoting guideline implementation.

\section{Background}

Carbapenem-resistant Enterobacteriaceae (CRE) are Gram-negative bacteria (e.g., Klebsiella sp. or Escherichia coli) that are resistant to most antibiotics. As of 2017, over 13,000 U.S. hospitalized patients had CRE, leading to 1,100 deaths and $\sim 130$ million in healthcare costs [1]. Carbapenemase-producing CRE (CP-CRE) refers to a subset of CRE whose enzymes break down carbapenems, rendering this last line of antibiotics ineffective [2]. CP-CRE infections are currently the main target of prevention efforts as they represent approximately $30 \%$ of all CRE, often can share carbapenemase genes with other bacteria leading to spread, and are associated with a greater than $50 \%$ mortality rate [3]; thus CRE/CP-CRE has been designated as an 'Urgent Threat,'CDC's highest risk level [1].

The Department of Veterans Affairs (VA) made CRE/CP-CRE prevention a national priority by developing and disseminating guidelines in 2015 to prevent the spread of these organisms [4]. The guidelines were updated in 2017 and 2019 to reflect the most current recommendations for CRE/CP-CRE prevention [5-6]. Key components of VA's 2015 guidelines/2017 directive include: 1) standardizing screening, identification, evaluation and reporting of CRE, including laboratory testing for CP-CRE; 2) increasing CRE surveillance; and 3) optimizing CRE infection prevention and control in acute care and VA nursing homes (community living centers, CLCs) including use of contact precautions, staff, and patient education materials, and strategies to track cases within and across VA facilities (e.g., interfacility transfer forms) [4-6]. While VA guidelines and directives form the basis of mandatory national policies, they do not provide additional funding or explicit directions/guidance on how to implement the guidelines at each VAMC. Implementing the new CRE guidelines/directive at VAMCs could be as simple as ensuring that all clinical staff attend in-service trainings. However, other recommendations might require purchasing new testing supplies or obtaining access to off-site VA or private sector labs to achieve timely CRE identification to inform clinical decision-making for patients infected with CRE. To better understand the challenges of consistent widespread guideline implementation across VA facilities, we collaborated with our Operations Partners in the VHA MDRO Program Office.

VA has been a national leader in implementing successful large-scale quality improvement infection control interventions [7-8]. As part of the MRSA Prevention Initiative, VA hired MDRO Program Coordinators (MPCs) (similar to an infection preventionist in the private sector) to support various Infection Control Program functions (e.g., MDRO surveillance, hand hygiene program, data collection, and reporting) [7]. We conducted a qualitative evaluation of the implementation of the 2015 guidelines/2017 directive using the Consolidated Framework for Implementation Research (CFIR) [9] to identify and understand the contextual factors and help characterize best practices and challenges that influenced CRE guideline implementation in VAMCs. 
This paper describes the results of analyses of semi-structured interviews to evaluate the implementation of the 2015 guidelines/2017 directive.

\section{Methods}

Semi-structured interviews were conducted at 29 VAMCs from October 2017 to August 2018 with MPCs, laboratory staff, infection preventionists, and physicians involved in implementing CRE guidelines at their facility. Interview questions focused on participants' perceptions of guideline implementation including dissemination, laboratory testing, staff attitudes and training, patient education, best practices, and technology support (e.g., medical record flag, laboratory report/template). Detailed notes were collected during phone interviews, and audio-recordings were consulted as needed to confirm their accuracy.

Study sites were selected to include the following factors: 1) geography (rural and urban), 2) VAMC size (e.g., large and small) and 3) VAMC CRE burden (high and low). Characteristics collected about VA facilities were obtained from the VA Office of Productivity, Efficiency, \& Staffing (OPES) Facility Complexity Model [10]. VA Corporate Data Warehouse (CDW), a relational database that captures all VA medical encounters, was used to identify the number of CRE positive clinical or surveillance cultures in 2016. CRE was defined as any culture with E. coli, Klebsiella spp., or Enterobacter spp. with nonsusceptibility to imipenem, meropenem, or doripenem [5]. Facility-level CRE burden was captured by dividing VAMCs into quartiles using the number of CRE positive cultures during 2016. CRE screening status was obtained from surveys completed by VA microbiology laboratory staff and MPCs collected as part of our larger study ("Support and Enhancement of VA Guidelines to Prevent the Spread of CRE ") [11-12].

We initially planned to use a stratified random sampling strategy. However, during the initial interviews, it became evident that while all sites were in the process of implementing the CRE guidelines, participants at sites with no CRE cases could only describe how they might hypothetically deal with CRE. Only sites with any CRE cases had concrete ideas and/or suggestions about improving screening, prevention, or management. To ensure that we collected the best available information on gaps and/or CRE practices, we oversampled facilities reporting active screening for CRE/CP-CRE and those with more CRE positive cultures.

Study team members involved in data collection and analysis included experts in implementation science and qualitative researchers (CCG, MLG), social scientists (CB, SR, AV) and infectious disease (ID) experts (epidemiologist (CE), pharmacist (KS), and physician (MF)). Our ID experts participated in the first 6 interviews $(14 \%)$ to ensure the quality of questioning and prompts. This study was approved by the Edward Hines Jr. VA Hospital Institutional Review Board as a Quality Improvement (QI) study [45 CFR 46.102(d)]. A copy of the Standards for Reporting Qualitative Research (SRQR; [13]) checklist is provided in Appendix 1.

\section{Analysis}


We conducted a consensus-based mixed deductive-inductive approach to coding participant responses from semi-structured interviews. Initial analysis of the detailed field notes from the first five interviews was conducted by the analysis team (CB, MG, CCG) and a codebook was developed, using CFIR constructs for guidance [9]. We developed our own codes, primarily to address emergent infection controlspecific issues [14]. Each team member initially coded each interview independently and then met as a group to reach consensus. To increase validity and reliability and refining content boundaries of codes and improving coding consistency, 12 percent of interviews were coded at regular team meetings (MLG, $\mathrm{CB}, \mathrm{CCG})$. The remaining interviews (88\%) were coded by two team members (CB, CCG), with coding discrepancies resolved with a third team member (MLG). Coding discrepancies involving clinical issues were resolved by ID experts (MF, KS, CE), who also provided clinical interpretations and classified responses as "best practices" if they described unique or innovative strategies. After each response was coded, it was then subsequently rated as positive, negative, or neutral using the criteria shown in Table 1. This is a modified version of the coding strategy used by Damschroder and colleagues (2013) [15].

Table 1

Criteria to rate constructs

\begin{tabular}{|ll|}
\hline+1 & $\cdot$ Positive influence on the site's ability to implement the guideline \\
\hline 0 & - No influence on the intervention/Describing the sites' usual practice \\
-1 & - Negative influence on the site's ability to implement the guideline \\
\hline
\end{tabular}

Damschroeder et al.'s (2013) sample size $(n=5)$ did not allow them to conduct a quantitative analysis of these constructs[15]. Our sample size was sufficient to conduct a quantitative analysis to examine factors associated with success in guideline implementation across all CFIR constructs and emergent open codes (codes that did not fall into one of the CFIR constructs). We compared the number of positive vs. negative responses for all CFIR constructs and open codes by whether the sites were actively screening for CRE (vs. non-screening) and by whether the site had any (vs. no) CRE positive cultures using Fisher's exact test. Due to the large number of comparisons, we decreased our alpha level to $p=$ 0.01 to adjust for multiple comparisons. Only significant results are reported (Table 2). 
Table 2

Quantitative analysis of CRE guideline implementation by CFIR Inner Setting constructs and open-code responses

\begin{tabular}{|c|c|c|c|c|c|c|}
\hline $\begin{array}{l}\text { CFIR variables } \\
\text { (definitions) }\end{array}$ & $\begin{array}{l}\text { By } \\
\text { variable }\end{array}$ & $\begin{array}{l}\text { Total } \\
\mathrm{N}(\%)\end{array}$ & $\begin{array}{l}\text { Positive } \\
{[+]} \\
\text { N (\%) }\end{array}$ & $\begin{array}{l}\text { Negative } \\
{[-]} \\
\mathbf{N}(\%)\end{array}$ & $\begin{array}{l}\text { P value } \\
\text { (Fisher's } \\
\text { exact } \\
\text { test) }\end{array}$ & Interpretation \\
\hline
\end{tabular}

Comparison of screening vs non-screening sites

$\begin{array}{lllll}\text { 1. Leadership } & \text { Not } & 22 & 15 & 7 \\ \text { Engagement } & \text { Screening } & (43.1) & (68.2)\end{array}$

$7 \quad 0.0015$

(31.8)

involvement and accountability of local leaders and managers with the guideline implementation $(\mathrm{n}=$ 51)

$\begin{array}{llll}\text { Screening } & 29 & 29 & 0 \\ & (56.9) & (100) & (0)\end{array}$

\begin{abstract}
Sites screening for CRE report more leadership involvement in implementing CRE policies compared to sites not screening for CRE: $100 \%$ vs. $68.2 \%$
\end{abstract}

Sites screening for CRE report CRE is treated as seriously as other HAls compared to sites not screening for CRE. $91.7 \%$ vs.
(44.4)

2
(57.1)
(91.7)

\section{$55.6 \%$}

Sites not screening for CRE report fewer available resources as compared to sites screening for CRE: $81.7 \%$ vs. $45.1 \%$ facilitate guideline implementation are available $(n=122)$

\begin{tabular}{|c|c|c|c|c|c|c|}
\hline $\begin{array}{l}\text { 4. CRE Reported } \\
\text { Incidence Episodes }\left({ }^{\star}\right)\end{array}$ & $\begin{array}{l}\text { Not } \\
\text { screening }\end{array}$ & $\begin{array}{l}19 \\
(57.6 \%)\end{array}$ & $\begin{array}{l}1 \\
(5.3 \%)\end{array}$ & $\begin{array}{l}18 \\
(94.7 \%)\end{array}$ & 0.005 & $\begin{array}{l}\text { Sites that } \\
\text { screen for CRE } \\
\text { reported more }\end{array}$ \\
\hline $\begin{array}{l}\text { Reported CRE } \\
\text { incidence }(Y / N)(n= \\
\text { 33) }\end{array}$ & & & & & & $\begin{array}{l}\text { CRE than sites } \\
\text { than non- } \\
\text { screening sites }\end{array}$ \\
\hline
\end{tabular}

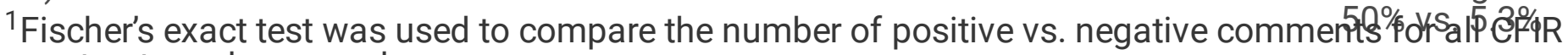
constructs and open codes

by screening vs. non-screening sites and any (vs. no) CRE positive cultures 


\begin{tabular}{|c|c|c|c|c|c|c|}
\hline $\begin{array}{l}\text { CFIR variables } \\
\text { (definitions) }\end{array}$ & $\begin{array}{l}\text { By } \\
\text { variable }\end{array}$ & $\begin{array}{l}\text { Total } \\
\mathrm{N}(\%)\end{array}$ & $\begin{array}{l}\text { Positive } \\
\text { [+] } \\
\mathbf{N}(\%)\end{array}$ & $\begin{array}{l}\text { Negative } \\
{[-]} \\
\text { N (\%) }\end{array}$ & $\begin{array}{l}\text { P value } \\
\text { (Fisher's } \\
\text { exact } \\
\text { test) }\end{array}$ & Interpretation \\
\hline
\end{tabular}

$\begin{array}{llll}\text { Screening } & 14 & 7 & 7 \\ & (42.4 \%) & (50 \%) & (50 \%)\end{array}$

\section{Comparison of sites with CRE cases vs. no CRE cases}

\begin{tabular}{|c|c|c|c|c|c|c|}
\hline $\begin{array}{l}\text { 1. Communication } \\
\text { Breakdown }\left(^{*}\right)\end{array}$ & CRE & $\begin{array}{l}27 \\
(50.9)\end{array}$ & $\begin{array}{l}27 \\
(100)\end{array}$ & $\begin{array}{l}0 \\
(0)\end{array}$ & \multirow[t]{2}{*}{0.02} & \multirow{2}{*}{$\begin{array}{l}\text { VAMCs with no } \\
\text { CRE cases } \\
\text { report more } \\
\text { communication } \\
\text { breakdown } \\
\text { than sites with } \\
\text { any CRE cases: } \\
100 \% \text { vs. } 80.8 \%\end{array}$} \\
\hline $\begin{array}{l}\text { communication or } \\
\text { breakdowns }(n=53)\end{array}$ & No CRE & $\begin{array}{l}26 \\
(49.1)\end{array}$ & $\begin{array}{l}21 \\
(80.8)\end{array}$ & $\begin{array}{l}5 \\
(19.2)\end{array}$ & & \\
\hline $\begin{array}{l}\text { 2. Access to } \\
\text { Knowledge and } \\
\text { Information }\end{array}$ & CRE & $\begin{array}{l}9 \\
(32.1)\end{array}$ & $\begin{array}{l}8 \\
(88.9)\end{array}$ & $\begin{array}{l}1 \\
(11.1)\end{array}$ & \multirow[t]{2}{*}{0.016} & \multirow{2}{*}{$\begin{array}{l}\text { Sites with any } \\
\text { CRE cases } \\
\text { report better } \\
\text { access to } \\
\text { knowledge and } \\
\text { information } \\
\text { than sites with } \\
\text { no CRE cases: } \\
88.9 \% \text { vs. } \\
36.8 \%\end{array}$} \\
\hline $\begin{array}{l}\text { Guideline or training } \\
\text { materials (e.g., } \\
\text { policies) locally } \\
\text { disseminated to } \\
\text { relevant stakeholders } \\
\text { at each facility }(n=25)\end{array}$ & No CRE & $\begin{array}{l}19 \\
(67.9 \%)\end{array}$ & $\begin{array}{l}7 \\
(36.8)\end{array}$ & $\begin{array}{l}12 \\
(63.2)\end{array}$ & & \\
\hline
\end{tabular}

${ }^{1}$ Fischer's exact test was used to compare the number of positive vs. negative comments for all CFIR constructs and open codes

by screening vs. non-screening sites and any (vs. no) CRE positive cultures

*Open codes

\section{Results}

\section{Participants}

We oversampled facilities reporting actively screening for CRE/CP-CRE (9/16) and those with more CRE positive cultures (Quartiles 1 and 2) as shown in Table 3. Forty-three interviews were conducted with microbiology laboratory staff (47\%), MPCs (35\%), infection control nurses (12\%), and physicians (6\%) as shown in Table 4.

\section{CFIR domains and constructs}

In Figure 1, we present the 841 interview coded responses categorized by CFIR domains related to system-wide CRE guideline implementation: 1$)$ Inner Setting ( $n=429,51 \%), 2)$ Implementation Process $(n=186,22.1 \%), 3)$ Intervention Characteristics $(n=133,15.9 \%), 4)$ Characteristics of Individuals $(n=49$, $5.8 \%)$, and 5) Outer Setting ( $n=44,5.2 \%)$ (see Appendix 2 for CFIR domain and construct definitions). 


\section{Quantitative results}

In Table 2, we show results of selected quantitative analyses comparing CFIR constructs and open codes by whether the sites were actively screening for CRE (vs. non-screening) and any (vs. no) CRE positive cultures. Our analysis identified the following constructs as being significantly associated with CRE screening or presence of CRE: Leadership Engagement, Relative Priority ("CRE is as important as other Hospital Acquired Infections (HAls)"), Available Resources (e.g., IT support, staffing), Team Communication (operationalized as communication breakdown), and Access to Knowledge \& Information (e.g., laboratory/clinical staff and patient/family educational material).

VAMCs that were actively screening for CRE reported significantly more leadership engagement in implementing CRE policies than those VAMCs that were not screening, $100 \%$ vs. $68.2 \%$, $(p=0.002)$. Sites screening for CRE reported that it was treated as seriously as other HAls (e.g., with Methicillin Resistant Staphylococcus aureus (MRSA)) when compared to sites not screening for CRE, 91.7\% vs. $55.6 \%$ $(p=0.01)$. Sites not screening for CRE were significantly more likely to report a lack of available resources (compared to sites screening for CRE) $81.7 \%$ vs. $45.1 \%$ ( $p<0.0001)$.

VAMCs with any CRE cases (vs. no CRE cases) were less likely to report team communication breakdowns than sites with no CRE, $100 \%$ vs. $80.8 \%$ ( $p=0.02)$. Sites with any CRE reported better access to knowledge and information compared with sites with no CRE, $88.9 \%$ vs. $36.8 \%(p=0.02)$.

\section{Qualitative Results}

Results of qualitative analyses include findings from representative responses coded by CFIR domains and constructs (Figure 1) as well as emergent open-coded responses identified during analysis, including responses categorized as "best practices" (defined as an innovative approach to implementation or guideline recommendations).

Within the Intervention Characteristics domain, responses categorized within the Intervention Source construct described which CRE guidelines participants reported using (e.g., VA, CDC, state), with most reporting using the VA's CRE guidelines. We coded responses that described adherence to CDC or state guidelines (not VA) as negative. Best practices examples involved sites adapting or modifying current MDRO policies to include recommendations from the VA CRE guideline.

Within the Outer Setting domain, Patient Needs \& Resources construct was defined as strategies and/or materials used to educate or engage Veterans and their families. Positive responses focused on the adequacy of and access to adequate educational materials to inform patients and their families about CRE (including the availability of materials in various languages and literacy levels). Negative responses described sites lacking knowledge of or access to handouts for educating laboratory and/or clinical staff and patients and families. An MPC described it this way:

It would be nice to have [more educational] materials [and staff release time for CRE-related training] $(M P C)$. 
Best practices described strategies to promote staff training, development of lab/clinical staff, and patient and family educational materials, including educational brochures targeting those with low literacy.

Within the Characteristics of Individuals domain, Self-Efficacy was defined as the participant's confidence in understanding and implementing CRE guidelines. Most participants' self-efficacy was high, reporting feeling "very" or "fully" confident in implementing the guidelines. The negative response described existing challenges in cohorting MRSA patients and being unsure of how to address CRE patients. Best practices included protected time for a dedicated person to monitor CRE at their VAMC.

Within the Implementation Process domain, the Formally Appointed Internal Implementation Leaders construct was defined as participant's existing infection control structure (units/departments/committee/resources) and/or laboratory testing of other MDROs. Negative responses centered on gaps in existing MDRO reporting strategies (e.g., overreliance on verbal vs. electronic communication). Best practices described a strong infection control structure based on good communication that enables sites to easily notify infection control team members of a CRE positive patient. Commercially available software (e.g., Theradoc $^{\circledR}$ ) was also described as facilitating staff communication.

Table 5 describes CFIR Inner Setting domains, definitions and representative positive, negative as well as best practices responses. Just over half of all coded responses addressed constructs within the Inner Setting domain (e.g., factors promoting successful CRE guideline implementation). Because these factors are potentially modifiable, and are important for addressing effective implementation, four of the top six

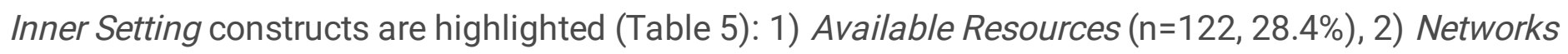
and Communication $(n=67,15.6 \%), 3)$ Leadership Engagement $(n=51,11.8 \%)$, and 4) Culture $(n=30$, $6.9 \%)$.

\section{Inner Setting Constructs}

\section{Available Resources:}

Successful implementation of the guidelines begins with stakeholders' perceptions of the immediacy of the problem. One interviewee described trying to implement the guidelines as very challenging in the absence of any CRE:

It's like waiting for it to snow in Florida. You're doing preparedness training [but] people don't listen and ... [they don't] think it's real. Because they haven't seen it ... People don't begin to be proficient unless they come in contact with it. [Laboratory Staff]

Participants at sites with CRE cases indicated that successful implementation of the CRE guidelines required access to and/or deployment of various resources (e.g., staffing, acquisition of new laboratory 
equipment to conduct polymerase chain reaction [PCR] testing, and/or information technology). One MPC described obtaining necessary resources in the following way:

[Our] Chief of Staff... did go to [the] chiefs of various services and said to them, you need to take the CRE screening seriously. Leadership certainly pushed all the physicians to implement the guidelines. If we ... had more positive CRE [cases], I'm positive they [leadership] will be on top of it. [MPC]

At sites where the guidelines were not fully implemented, participants described inefficient or overly complicated processes for obtaining new laboratory equipment and/or addressing staff turnover, insufficient contact isolation rooms, and/or dependence on unreliable strategies (e.g., word-of-mouth) for communicating patient CRE status.

Best practices for the Available Resources construct included strategies to enhance dissemination of CRE educational materials to providers (e.g., posters and train-the-trainer in-services), timely access to laboratory reports to inform clinical decision-making, and more formal strategies to systematically communicate patients' CRE test results (positive or negative) to all relevant parties (e.g., admission/discharge templates in the electronic health record [EHR]).

To confirm CP-CRE, all VAMCs who did not have the available equipment were required to purchase new laboratory equipment or to identify an outside laboratory with PCR-testing capacity. Once a potential case of CP-CRE was identified, participants described ongoing monitoring of confirmation test results of CPCRE and developing strategies to ensure adequate isolation space to keep the identified individual in contact precautions until CP-CRE status was confirmed.

\section{Networks and Communication:}

Coded responses described positive and negative aspects regarding CRE guideline dissemination. Sites reporting good communication described adapting a robust pre-existing infection control infrastructure to successfully address CRE. Other sites cited overly complicated and/or hierarchical bureaucracy that hindered dissemination, contributing to delays in development or approval of local policies as impeding CRE guideline implementation. Best practices for this construct included employing strategies, tools, and innovations to facilitate local guideline implementation. One such strategy was to include CRE in existing MDRO policies versus developing a CRE specific policy, which often delayed guideline implementation. Participants most commonly reported learning about the VA CRE Guidelines through one or more national, regional, and local communication mechanisms, including hospital and discipline-specific (e.g., laboratory, infectious disease, pharmacy) email listservs. Guidelines were also discussed and disseminated at local in-service trainings, new employee orientation, and/or ad hoc trainings (e.g., daily patient rounds), as well as during local interdisciplinary committee meetings (e.g., antibiotic stewardship committee meetings).

\section{Leadership Engagement:}


Leadership engagement was defined in terms of local leaders' commitment to, involvement in, and accountability for CRE guideline implementation. Sites successfully implementing the CRE guidelines commonly cited local (VAMC, laboratory, and/or Infection Control [IC]) leadership engagement in facilitating timely procurement of laboratory testing equipment. Negative responses described local (VAMC, laboratory and/or IC) leadership as "uninvolved" in guideline implementation and/or unwilling to address adequate isolation space, staffing to conduct CRE testing, release time for staff education, or funding to purchase laboratory equipment. Best practices described active leadership engagement in guideline implementation and participation by all relevant clinical and laboratory staff.

\section{Culture:}

Culture responses were classified as "positive" when they described strong local infection control practices, a proactive approach to CRE prevention and/or management and/or heightened awareness of infection control and prevention for emerging pathogens (e.g., active screening for CRE in high-risk patients or a plan to incorporate active screening into current practices if or when CRE incidence increased). "Negative" culture responses described local knowledge about CRE as lacking and/or poor staff compliance with hand hygiene and/or use of personal protective equipment (PPE) (gowns or gloves):

It's very challenging, I've been here a year. When I do environmental rounding and corrections, if someone is not [using] PPE for example, [I get] a lot of push back. I think [staff] think CRE is serious, because I'll go talk to them face-to-face, if someone has a history of it. But MRSA, isn't treated as seriously. [MPC]

Best practices examples for culture addressed interdisciplinary collaboration, education, and/or crosstraining of laboratory staff on microbiology/ infection control practices.

Table 3

Facility Characteristics by CRE Incident (Quartiles)

\begin{tabular}{|lllll|}
\hline CRE *Quartiles $\rightarrow$ & Q1 & Q2 & Q3 & Q4 \\
\hline CRE Positive Cultures (range, $N)$ & $16-239$ (mean = 46) & $7-15$ & $3-6$ & $0-2$ \\
\hline Sites reporting Screening $(\mathrm{N}=9)$ & 5 & 1 & 3 & 0 \\
\hline Sites participating in interviews $(\mathrm{N}=28)$ & 11 & 7 & 7 & 3 \\
\hline Interviewees $(\mathrm{N}=43)$ & 22 & 8 & 7 & 6 \\
\hline $\begin{array}{l}\text { *Facility-level CRE burden was calculated by dividing VAMCs into quartiles using the number of CRE } \\
\text { positive cultures during 2016 }(\mathrm{N}=141) \text {. }\end{array}$ & & & \\
\hline
\end{tabular}


Table 4

Interviewee Characteristics

\begin{tabular}{|c|c|}
\hline Individuals Interviewed & Total Participants $(\mathrm{N}=43)$ \\
\hline \multicolumn{2}{|l|}{ Gender } \\
\hline Male & 11 \\
\hline Female & 32 \\
\hline Mean Years at VA (SD)* & $12.34(10.35)$ \\
\hline Mean Years in Current Position (SD)** & $6.26(6.11)$ \\
\hline \multicolumn{2}{|l|}{ Occupation } \\
\hline Microbiology Laboratory Staff & 20 \\
\hline Multidrug-Resistant Organisms Prevention Program Coordinator (MPC) & 15 \\
\hline Infection Control Nurse & 5 \\
\hline Infection Control Physician & 3 \\
\hline *Missing 1 observation & \\
\hline
\end{tabular}


Table 5

Representative positive, negative, and best practices responses for CFIR Inner Setting domains for CRE guideline implementation

\section{CFIR Domains Representative Responses}

$\begin{array}{llll}\text { 1. Available } & \text { Positive } & \text { Negative } & \text { Best Practices } \\ \text { Resources } & \end{array}$

Definition:

Money, equipment, testing supplies, training, education, isolation space, staff time, IT support, and previous workarounds to facilitate guideline implementation are available

\section{IT support and staffing:}

(...) we have a CPRS

[discharge] template.

[CRE status] would be communicated in the template and the nurses [also] call the accepting facility and give a [verbal] report. [MPC]

Timely lab reporting.

The [CRE testing] cartridges last about 46 months, (...) and if I only get 3 [CRE cases] within those 6 months, then I have to throw the other 7 cartridges away. [By comparison, the University lab can provide] results in] ... about 24 hours. [Laboratory]

\section{Lab testing equipment \& training:}

[New lab equipment has to be purchased and time has to be allocated for staff to be trained and be proficient.] It's hard to add new testing in the VA. [Laboratory]

\section{Staffing:}

[We] had a lot of [staff] turnover in infection control for a long time, and when you have less experienced with] less training, then that's a challenge.

[Infection Control Staff]

We just don't have the staff [to implement CRE screening] right now.

\section{[MPC]}

\section{Educational \\ materials/Staffing.}

It would be nice to have [more educational] materials [and staff release time for CRErelated training]. I tried ... to do an in-service every 30 minutes and [only] had 5 people that day. [MPC]

Isolation beds:

[Our] spinal cord [unit] is a problem because they [only] have four bed room[s] [individual isolation rooms are not available]. That's an infection control issue if someone is [CRE] positive. [MPC]

IT support.
Staff training

workaround:

[In addition to] unitbased training, [we] developed poster boards [for] the Infection Control week. After [the] initial training, physicians were still ignoring and bypassing order sets. [So] posters [were hung] in meeting rooms and any rooms where physicians did their documentations. They finally are getting numbers that are representative. [Infectious Disease Chief]

\section{Training and materials.}

The acute care people do not think it's important to have a full class. ... I do a train-thetrainer session [now]. [MPC]

\section{Lab reporting.}

But since [our lab] system was updated with the new isolates and packages, we are now able to select the CRE organism. We are doing active surveillance and Infection Control developed a list of high-risk patients and 
The handoff communication is verbal and the transfer note doesn't [routinely address the patient's] isolation status. When I call the new unit, [unless] they check their Theradoc $\AA_{\text {.... they won't }}$ know. [MPC] recommendations for screening from the guidelines. [Laboratory]

If a [CRE+] patient is... flagged from a previous encounter, ...they are automatically put in isolation on admission [based on] the Theradoc ${ }^{\circledR}$ documentation. [Laboratory]

2. Networks \&
Communication
Definition: Local
infrastructure
for national
guideline or
training
material
dissemination
(e.g., CRE-
related
conference calls
to train staff).

Representative Responses Positive

[Our] electronic communication

includes... a daily print out... an email group ... [for] asking questions and vice versa ... [and] hard copies of [overnight] test results. There may [also] be a phone call or a voicemail. It's [a] multiprong approach. ... We have pamphlets online that they [staff] can print if we [Infection Control] aren't around. We try to coach them to be selfsufficient. [Infection Control Nurse]

[Guideline information is disseminated] through [the] MDRO group, ... front office ... [and] the VISN. [Staff] attend [the] Antimicrobial Stewardship Program interdisciplinary committee meeting, ... staff meetings, ... [and receive] email from the IP doctor.

[MPC]

\section{Negative Best Practices}

There is a lag in the policy being implemented once published. [I] didn't get [timely MPC-level] access to the [MDR Office] communications, emails, and calls. [Infection Control Manager]

There were a lot of delays here, $\ldots$ outdated policies and possibly didn't rise to the top as priorities], [causing] a lag in communications... [So, guideline] issues haven't been brought up [to VAMC and VISN] Infection Control yet.

[Infection Control Manager]
I also created an [CP-CRE] algorithm

... [that] informed everyone ... who to test, what to do, and when to contact Infectious Disease. There is also a binder on every unit where everything sits. [MPC]

I go to [the units] and make sure they have all signs up, ... [and that] they have ... [participated in a CRE] in-service. I asked them to educate the patient ... or l'll do it with them if they aren't comfortable. I also talk to the attending and resident physician as well. Some don't understand the need for isolation, so it's important we speak to everyone. [MPC]
3. Leadership Engagement

Definition:
Representative Responses

$\begin{array}{lll}\text { Positive } & \text { Negative } & \text { Best } \\ & \text { Practices }\end{array}$




\begin{tabular}{|c|c|c|c|}
\hline $\begin{array}{l}\text { Commitment, } \\
\text { involvement } \\
\text { and } \\
\text { accountability } \\
\text { of local leaders } \\
\text { and managers } \\
\text { with the } \\
\text { guideline } \\
\text { implementation. }\end{array}$ & $\begin{array}{l}\text { [If we tell leadership } \\
\text { what's needed], they'll } \\
\text { say okay and get it for } \\
\text { us. If we started seeing } \\
\text { more CREs, and we need } \\
\text { [new testing equipment], } \\
\text { they'll find the money to } \\
\text { support it. } \\
\text { [Laboratory] } \\
\text { Laboratory [leadership } \\
\text { handles all] equipment } \\
\text { needs ... and any new } \\
\text { assays. } \\
\text { [Infection Control Staff] } \\
\text { [Based on previous } \\
\text { experience,] we go to } \\
\text { [leadership] saying we } \\
\text { need ... [lab equipment] } \\
\text { and they'll say okay and } \\
\text { get it for us. [Laboratory] }\end{array}$ & $\begin{array}{l}\text { [Leadership] } \\
\text { allowed us } \\
\text { to do a } \\
\text { CPRS flag } \\
\text { and ... to } \\
\text { send out } \\
\text { confirmation } \\
\text { testing and } \\
\text {... set up } \\
\text { send out } \\
\text { protocols. } \\
\text { However, it } \\
\text { takes a long } \\
\text { time to get } \\
\text { this much } \\
\text { done. [MPC] }\end{array}$ & $\begin{array}{l}\text { [Our] Medical } \\
\text { Director is } \\
\text { also very } \\
\text { involved in } \\
\text { Infection } \\
\text { Control and } \\
\text { Infectious } \\
\text { Disease... } \\
\text { [and] works } \\
\text { with [us] on } \\
\text { projects that } \\
\text { arise. } \\
\text { [MPC] } \\
\text { We've } \\
\text { implemented } \\
\text { a great } \\
\text { [computer] } \\
\text { tool ... for } \\
\text { screening } \\
\text { admissions } \\
\text { for [CRE] risk } \\
\text { factors and } \\
\text { [are] } \\
\text { implementing } \\
\text {.. swabbing } \\
\text { for those with } \\
\text { risk factors } \\
\text { (e.g., } \\
\text { international } \\
\text { travel, organ } \\
\text { transplant } \\
\text { recipients). }\end{array}$ \\
\hline & & & \\
\hline
\end{tabular}

4. Culture

Definition:

Infection

Control policies

already

implemented

locally or in anticipation of new MDROs.
Representative responses

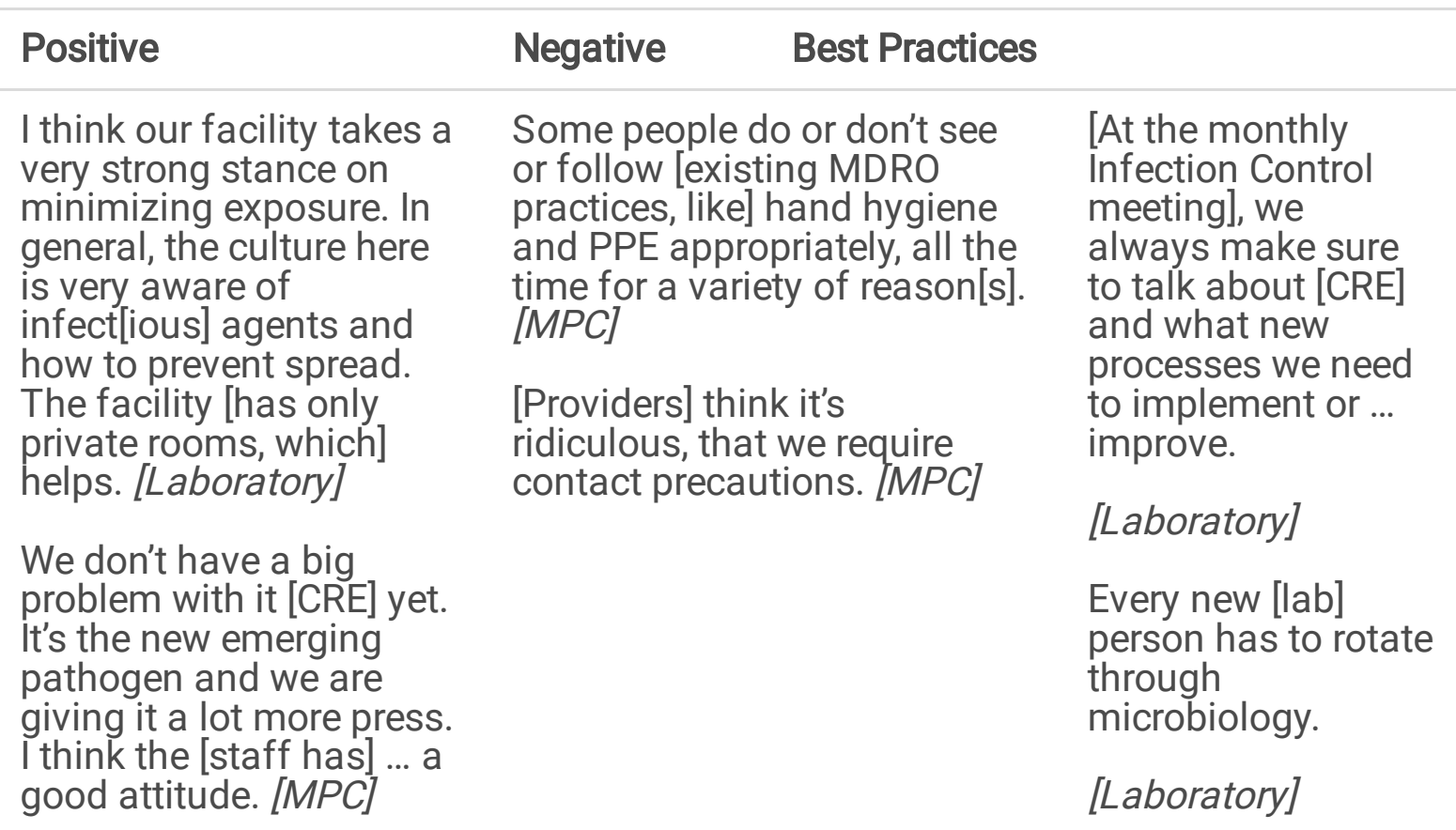


We've done an

excellent job with

MDROs at the

facility, people are

very cooperative

and enthusiastic.

[Our] MRSA [rate] is

dropping rapidly.

People are really

working hard .... with

Infection Control ...

to clean rooms, ...

come to the

Infection Control

meetings, and there

[are] always

questions. The

[Infectious Disease]

Chief does a lunch

and learn... every

month. He answers

any questions

about infection

control.

[Laboratory]

\section{Discussion}

Healthcare-associated infections are common, with greater than 1 million occurring annually across the U.S. healthcare system, representing an ongoing threat to patient safety [16]. In 2020, in comparison with other MDROs, CRE remains relatively uncommon in VAMCs. A subset of CRE, CP-CRE, are particularly worrisome as they may be responsible for increasing CRE spread in the U.S. [1] and mortality rates due to CP-CRE remain high [17-19]. Thus, VA is implementing guidelines in all acute care and skilled nursing facilities to prevent further spread of CRE. Nearly all interviewees in our larger study reported using VA CRE/CP-CRE guidelines, most with high confidence (79\%) [12].

Effective CRE identification, prevention, and treatment requires ongoing collaboration between clinical, microbiology, infection prevention, antimicrobial stewardship, and infectious diseases specialists.

Evidence suggests that prevention can be strengthened by using standardized tools that facilitate effective communication as well as identify gaps in policies/ procedures (e.g., electronic admission and discharge templates, patient flags, protocols, and strong IC organizational culture) [20-24].

Based on our analyses, CRE incidence plays an important role in increasing awareness and willingness to take steps to fully implement the guideline. While VA actively screens all new admissions for other MDROs (e.g., MRSA), CRE screening remains uncommon at most VA facilities. In the absence of any CRE incidence at a given facility, guideline implementation was described as very challenging. During our initial interviews, it became clear that the experience of even one CRE case strongly increased staff perceptions of the importance of implementing the guidelines and overall priorities for CRE at their 
facility. This was confirmed in our quantitative analyses, where having any CRE positive cultures was associated with helping sites to identify and address potential gaps in existing policies and/or procedures.

CRE prevention and management builds on strong infection control practices and facility culture. Our results indicate that existing MDRO policies and practices may need to be modified to address CREspecific issues of identification, prevention, and/or management. For example, available resources were identified as crucial for the successful implementation of the CRE guidelines. Even sites that reported success in implementing the guidelines requested more standardized CRE prevention and management educational materials for: 1) laboratory staff, 2) clinical staff, and 3) patient and family members. Research supports the idea that for infection prevention practices to be successful, healthcare workers must receive ongoing infection prevention specific education [25-30]. Participants also identified the need for training materials for new and existing clinical staff and dedicated training time to ensure that all clinicians understood relevant risk factors, prevention, and management strategies for patients with CRE. The need for educational materials, especially for patients and families, is well established within the healthcare setting [31]. In particular, requests were made for CRE-specific patient/family education materials for staff to facilitate obtaining consent to collect rectal swabs. VA MDRO Program Office has provided virtual training, staff and patient/family educational resources, and shared documents via SharePoint.

The empirical evidence supporting the efficacy of education, by itself, remains weak. Research suggests that education may be more effective in promoting behavior change when combined with other strategies [32-33]. We and others (Ellis et al., 2005) found many other factors, including culture and environment, management structures, and resources are also needed for an organization to successfully implement guidelines [34].

We previously found that strong leadership support was essential and specifically identified as a facilitator for successful implementation of the MRSA infection prevention guidelines in Spinal Cord Injury and Disorders settings across VAMCs [35]. These findings also highlighted the importance of strong local leadership, particularly VAMC laboratory and/or infection control leadership, on the success of CRE guideline implementation. Leadership is key in providing adequate resources [25] and helping shape positive workplace/facility culture [33, 36-37]. These current results emphasize the importance of local leadership's role in promoting positive facility culture which may include healthcare worker's heightened awareness of infection control and prevention for emerging pathogens, facilitating successful implementation of the CRE guidelines.

At the time the guidelines were disseminated, the MDRO Program Office held multiple online training sessions, including real-time question and answer sessions as part of their regularly scheduled monthly MPC calls. Our interview guide included questions about CRE guideline dissemination, in-person conferences and education/training materials. While we did not specifically ask about perceptions of the ongoing MDRO Program Office training program or access to training materials, some participants, 
primarily MPCs, described a lack knowledge of the availability of training and educational resources to support local guideline implementation. It is unclear how local leadership support and dedicated resources are related to MPC's percieved lack of knowledge of available national resources. However, issues of staff turnover, VA's shift from large in-person conferences to virtual training, context, and culture likely contribute.

Changes in clinical practice rely on both the nature and strength of the evidence as well as the setting in which the practice is to be placed, including the way that the process is facilitated [38-39]. Further, implementation interventions generally target patient-, provider-, and/or system-level behavior change [32]. Responses classified as contextual factors described a variety of factors associated with successful implementation of the CRE guideline. Some contextual factors are easier to change than others. For example, increasing access to isolation rooms for CRE-positive patients is challenging to address, especially in older hospitals, with multi-bed rooms. Less concrete factors, like culture, can be more open to change when a critical mass of individuals perceive an immediate threat. This threat can then push people to very quickly change what might have previously been seen as unchangeable.

One of the most urgent contextual factors within the CFIR networks and communication construct was the need for proactive, consistent information exchange about patient CRE status across all clinical providers and settings. Effective communication can interrupt CRE spread, highlighting the necessity of information technology to support reporting of patient CRE/CP-CRE status within and between settings. Prompt and accurate identification of and communication about patient CRE status at admission and discharge is vital for improving infection control outcomes [20; 40-43]. Our findings support the potential for standardized laboratory, admission, and discharge templates to improve and communicate patient CRE status.

\section{Limitations}

This study was conducted in VA, and perceptions and practices may be different in the private sector or non-US hospitals. In addition, we used a cross-sectional study design during a dynamic period of change in practices to address CRE. Many of these practices and perceptions may likely have been addressed or changed since our interviews were conducted.

\section{Conclusions}

CRE/CP-CRE incidence is increasing and it has spread throughout the U.S., with the potential to spread within all acute and long term care facilities. Controlling CRE spread and improving patient outcomes requires timely and reliable detection and identification methods, followed by effective antibiotic treatment and implementation of enhanced infection control practices. Sites successfully implementing CRE guidelines described having access to available resources, effective communication tools, and strategies, engaged leadership, as well as a strong infection control infrastructure. Further, CRE/CP-CRE prevention requires a comprehensive multi-faceted approach to interrupt its spread including access to adequate resources and communication capabilities. We will continue to collaborate with VHA's MDRO 
Program Office to understand participants' perceived lack of knowledge of available training and educational resources and work to develop targeted implementation strategies to bridge gaps to strengthen VA CRE/CP-CRE prevention efforts.

\section{List Of Abbreviations}

VHA: Veterans Health Administration

VAMCs: Veteran Affairs Medical Centers

HAls: health-care acquired infections

VA: Veteran's Affairs

MDRO: multi-drug resistant organisms

MPCs: Multi-Drug Resistant Organism Program Coordinators

CRE: Carbapenem-resistant Enterobacteriaceae

CP-CRE: Carbapenamase-Producing CRE

\section{Declarations}

Ethics approval and consent to participate: The study was approved by Edward Hines Jr. VA Hospital Institutional Review Board as a Quality Improvement (QI) study [45 CFR 46.102(d)].

Consent for publication: Not Applicable

Availability of data and materials: The datasets generated and/or analyzed during the current study are not publicly available due to participant privacy but are available from the corresponding author on reasonable request.

Competing interests: The authors declare that they have no competing interests.

Funding: Funding support was provided by the Quality Enhancement Research Initiative (QUERI) Award QUE 15-269.

Authors' contributions: MG, CRB were responsible for data collection; MG, CRB, CCG had full access to all of the data in the study and take responsibility for the integrity of the data and the accuracy of the data analysis. CTE, ENP, and MR were responsible for the concept and design. CCG, MG, CRB, AV, SR, LP, MF, $\mathrm{KS}, \mathrm{CTE}$ were responsible for analysis and interpretation of the data. CCG, MG, LP performed statistical analysis. CCG and MG drafted the manuscript. All authors provided critical revision of the manuscript for important intellectual content. Funding was obtained by CTE, ENP, MR. 
Acknowledgements: We are grateful to the MDRO Program Office and the infection control and prevention staff and mircobiology laboratory staff who shared their perceptions of VA CRE guideline implementation.

\section{References}

1. Centers for Disease Control and Prevention. Antibiotic Resistance Threats in the United States, 2019. Atlanta, GA: U.S. Department of Health and Human Services, CDC; 2019.

2. Patel G, Huprikar S, Factor SH, Jenkins SG, Calfee DP. Outcomes of carbapenem-resistant Klebsiella pneumoniae infection and the impact of antimicrobial and adjunctive therapies. Infect Control Hosp Epidemiol. 2008;29(12):1099-1106.

3. Centers for Disease Control and Prevention. CRE technical information. https://www.cdc.gov/hai/organisms/cre/technical-info.html Last accessed June 30,/2020.

4. VHA National Infectious Disease Service, MDRO Prevention Office. 2015 Guideline for Control of Carbapenem-Resistant Enterobacteriaceae (CRE).

5. VHA National Infectious Disease Service, MDRO Prevention Office. 2017 Guideline for Control of Carbapenemase Producing-Carbapenem Resistant Enterobacteriaceae (CPCRE).

6. VHA 2019 Toolkit for Control of Carbapenemase-Producing, Carbapenem-Resistant

Enterobacteriaceae (CP-CRE); VHA MDRO Prevention Office, 11/22/19; http://vaww.mrsa.va.gov/Carbapenem_resistant_Enterobacteriaceae_CRE.asp

7. MRSA Initiative S. Department of Veterans Affairs VHA. Methicillin-resistant Staphylococcus Aureus (MRSA) initiative, VHA Directive 2007-002. 2007.

[http://www1.va.gov/vhapublications/ViewPublication.asp?pub_ID=2163]. Last accessed June 30, 2020.

8. Jones M, Nielson C, Gupta K, Khader K, Evans M. Collateral benefit of screening patients for methicillin-resistant Staphylococcus aureus at hospital admission: Isolation of patients with multidrug-resistant gram-negative bacteria. Am J Infect Control. 2015; 43:31-34.

9. Damschroder LJ, Aron DC, Keith RE, Kirsh SR, Alexander JA, Lowery JC. Fosteringimplementation of health services research findings into practice: a consolidated framework for advancing implementation science. Implement Sci. 2009; 5:50.

11. Fitzpatrick MA, Suda KJ, Ramanathan S, et al. Laboratory practices for identification and reporting of carbapenem-resistant Enterobacteriaceae in Department of Veterans Affairs facilities. Infect Control Hosp Epidemiol. 2019; 40(4): 463-66. 
12. Ramanathan S, Suda KJ, Fitzpatrick MA, Guihan M, Goedken CC, Safdar N, Evans M, Jones M, Pfeiffer CD, Perencevich EN, Rubin M, Evans CT, QUERI CARRIAGE Program. A survey of Infection Control Strategies for Carbapenem-resistant Enterobacteriaceae in Department of Veterans Affairs Facilities. Infect Control Hosp Epidemiol. 2020; (in press).

13. O'Brien BC, Harris IB, Beckman TJ, Reed DA, Cook DA. Standards for reporting qualitative research: a synthesis of recommendations. Academic Medicine, Vol. 89, No. 9 / Sept 2014

14. Ryan GW, Bernard RH. Techniques to Identify Themes. Field Methods. 2003; 15:1:85-109.

15. Damschroder et al., Evaluation of a large-scale weight management program using the consolidated framework for implementation research. Implement Sci. 2013; 8:51.

16. AHRQ's Healthcare-Associated Infections Program. August 2019. Agency for Healthcare

17. Chen WK, Yang Y, Tan BH. Increased Mortality Among Carbapenemase-Producing CarbapenemResistant Carriers Who Developed Isolates of Another Genotype. Open Forum Infect Dis. 2019 Feb $1 ; 6(2)$ :ofz006.

18. Vital signs: carbapenem-resistant Enterobacteriaceae. Centers for Disease Control and Prevention (CDC). MMWR Morb Mort Wkly Rep. 2013;62(9):165-170.

19. Martin A, Fahrbach K, Zhao Q, Lodise T. Association Between Carbapenem Resistance and Mortality Among Adult, Hospitalized Patients With Serious Infections Due to Enterobacteriaceae: Results of a Systematic Literature Review and Meta-analysis. Open Forum Infect Dis. 2018 Jun 28;5(7):ofy150.

20. Lee BY, Bartsch SM, Wong KF, et al. Simulation show hospitals that cooperate on infection control obtain better results than hospitals acting alone. Health Affairs. 2012; 31(10): 2295-303.

21. Huang SS, Avery TR, Song Y, et al. Quantifying interhospital patient sharing as a mechanism for infectious disease spread. Infect Control Hosp Epidemiol. 2011; 31(11): 1160-69.

22. Foote MMK, Styles TS, Quinn CL. Assessment of Hospital Emergency Department Response to Potentially Infectious Diseases Using Unannounced Mystery Patient Drills. New York City, 2016. MMWR Morb Mort Wkly Rep. 2017; Sep;15;66(36):945-949.

23. Ali M, Williams MD.J Community Health. Notice Mystery Patient Drills to Assess Emergency Preparedness for Infectious Diseases at Community Health Centers in New York City, 2015-2016. 2019; Apr;44(2):387-394.

30. Siegel JD, Rhinehart E, Jackson M, Chiarello L, and Healthcare Infection Control Practices Advisory Committee. Management of Multidrug-Resistant Organisms in Healthcare Settings, 2006. Am J Infect Control. 2007; Dec;35 (10 Suppl 2):S165-93. 
31. Behar-Horenstein LS, Guin P, Gamble K, Hurlock G, Leclear E, Philipose M, Weldon J. Improving patient care through patient-family education programs. Hospital Topics. 2005;83(1):21-27.

32. Kirchner E, Ritchie M, Pitcock A, Parker LE, Curran GM, Fortney JC. Outcomes of a partnered facilitation strategy to implement primary care-mental health. J Gen Intern Med. 2014;29 suppl 4:S904-12. 29.

33. Zingg W, Holmes A, Dettenkofer M, Goetting T, Secci F, Clack L, Allegranzi B, Magiorakos AP, Pittet D. Hospital organisation, management, and structure for prevention of health care-associated infection: a systematic review and expert consensus. The Lancet Infectious Diseases. 2015; Feb; 15(2):212224.

34. Ellis I, Howard P, Larson A, Robertson J. From workshop to work practice: an exploration of context and facilitation in the development of evidence-based practice. Worldviews Evid Based Nurs. 2005;2(2):84-93.

35. Balbale S, Hill JN, Guihan M, Hogan T, Cameron KA, Goldstein B, Evans CT. Evaluating implementation of methicillin-resistant Staphylococcus aureus (MRSA) prevention guidelines in spinal cord injury centers using PARIHS framework: a mixed methods study. Implement Sci. 2015; Sep;10:130.

36. Dodek P, Cahill NE, Heyland DK. Implementation of Clinical Practice Guidelines. J Parenter Enteral Nutr. Nov-Dec 2010;34(6):669-74.

37. Krein SL, Damschroder LJ, Kowalski CP, Forman J, HoferTP, Saint S. The influence of organizational context on quality improvement and patient safety efforts in infection prevention: A multi-center qualitative study. Social Science \& Medicine. 2010; Nov;7(9):1692-1701.

38. Gifford W, Davies B, Edwards N, Griffin P, Lybanon V. Managerial leadership for nurses' use of research evidence: an integrative review of the literature. Worldviews Evid Based Nurs. 2007;4(3):126-145.

39. Rycroft-Malone J, Seers K, Titchen A, Harvey G, Kitson A, McCormack B. What counts as evidence in evidence based practice? J Adv Nurs. 2004;47(1):81-90.

40. Huang SS, Avery TR, Song Y, Elkins KR, Nguyen CC, Nutter SK, et al. Quantifying interhospital patient sharing as a mechanism for infectious disease spread. Infect Control Hosp Epidemiol. 2011; 31(11): 1160-69.

41. Pfeiffer CD, Cunningham MG, Poissant T, Furano JP, Townes JM, Leitz A, Thomas A, Buser G, Arao RF, Bledavs ZG. Establishment of a statewide network for carbapenem-resistant enterobacteriaceae prevention in a low-incidence region. Infect Control Hosp Epidemiol. 2014; 35(4): 356-61. 
42. Shimasaki T, Segreti J, Tomich A, Kim J, Hayden MK, Ling MY. Active screening and interfacility communication of carbapenem-resistant Enterobacteriaceae (CRE) in a tertiary-care hospital. Infect Control Hosp Epidemiol. 2018; Sep;39(9): 1058-62.

43. Trepanier P, Mallard K, Muenier D, Pike R, Brown D Ashby JP, Donaldson H, Awad-El Kariem FM, Balakrishnan I, Cubbon M, Chadwick PR, Doughton M, Doughton R, Hardiman F, Jarvey G, et al. Carbapenem-producing Enterobacteriaceae in the UK: a national study (EuSCAPE-UK) on prevalence, incidence, laboratory detection methods and infection control measures. J Antimicrob Chemother. 2017; 72(2): 596-603.

\section{Figures}

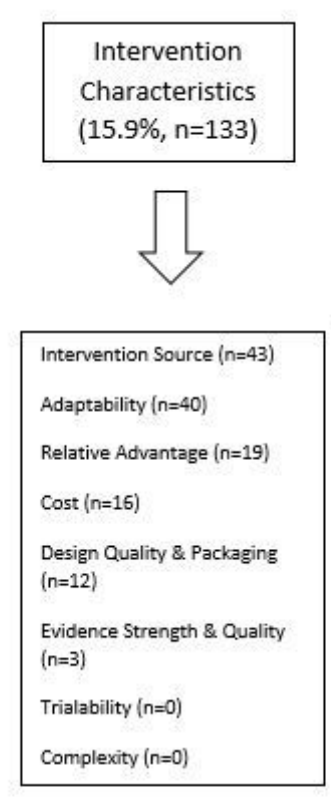

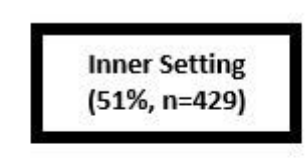

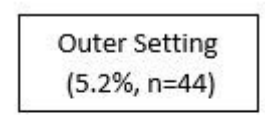

\section{Available Resources ( $n=122$ )}

Networks \& Communication ( $n=67$ )

Leadership Engagement ( $n=51$ )

Relative Priority $(n=43)$

Structural Characteristics ( $n=37$ )

Culture $(n=30)$

Access to Knowledge \& information

( $n=28)$

Readiness for Implementation ( $n=12$ )

Tension for Change ( $n=3$ )

Implementation Climate ( $n=1)$

Compatibility $(n=0)$

Organizational Incentives \& Rewards $(n=0)$

Goals \& Feedback $(n=0)$
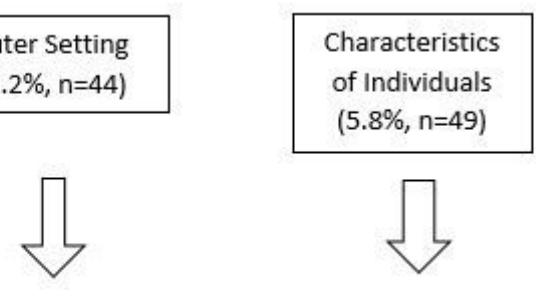

Patient Needs \& Resources ( $n=29$ )

External Policy \& Incentives $(n=15)$

Cosmopolitanism ( $n=0$ )

Peer Pressure $(n=0)$

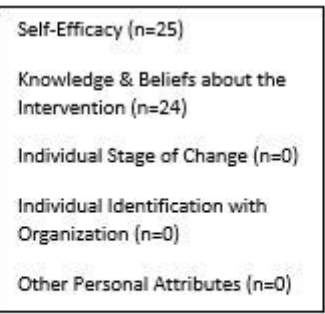

Self-Efficacy $(n=25)$

Knowledge \& Beliefs about the Intervention $(n=24)$

Individual Stage of Change $(n=0)$

Individual Identification with

Other Personal Attributes $(n=0)$
Implementation Process

(22.1\%, $n=186)$

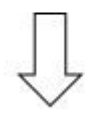

Formally Appointed Internal Implementation Leaders $(n=54)$

Planning ( $n=51$ )

Champions ( $n=34)$

Reflecting \& Evaluation $(n=21)$

Engaging ( $n=14$ )

External Change Agent $(n=9)$

\section{Figure 1}

Frequency of Responses by CFIR Implementation Domains \& Constructs

\section{Supplementary Files}

This is a list of supplementary files associated with this preprint. Click to download.

- SQSRFinalAppendix181020.docx

- Appendix2Final.docx 
Page 25/25 